\title{
Temporal and spatial effect of air pollution on hospital admissions for myocardial infarction: a case-crossover study
}

\author{
Xiaoxiao Liu PhD, Stefania Bertazzon PhD, Paul J. Villeneuve PhD, Markey Johnson PhD, Dave Stieb MD, \\ Stephanie Coward PhD, Divine Tanyingoh MSc, Joseph W. Windsor PhD, Fox Underwood MSc, \\ Michael D. Hill MD, Doreen Rabi MD, William A. Ghali MD, Stephen B. Wilton MD, \\ Matthew T. James MD, Michelle Graham MD, M. Sean McMurtry MD, Gilaad G. Kaplan MD
}

\section{Abstract}

Background: In studies showing associations between ambient air pollution and myocardial infarction (MI), data have been lacking on the inherent spatial variability of air pollution. The aim of this study was to determine whether the long-term spatial distribution of air pollution influences short-term temporal associations between air pollution and admission to hospital for MI.

Methods: We identified adults living in Calgary who were admitted to hospital for an MI between 2004 and 2012. We evaluated associations between short-term exposure to air pollution (ozone $\left[\mathrm{O}_{3}\right]$, nitrogen dioxide $\left[\mathrm{NO}_{2}\right]$, sulfur dioxide $\left[\mathrm{SO}_{2}\right]$, carbon monoxide $[\mathrm{CO}]$, particulate matter $<10 \mu \mathrm{m}$ in diameter $\left[\mathrm{PM}_{10}\right]$ and particulate matter $<2.5 \mu \mathrm{m}$ in diameter $\left[\mathrm{PM}_{2.5}\right]$ ), and hospital admissions for MI using a timestratified, case-crossover study design. Air Quality Health Index (AQHI) scores were calculated from a composition of $\mathrm{O}_{3}, \mathrm{NO}_{2}$ and $\mathrm{PM}_{2.5}$. Conditional logistic regression models were stratified by low, medium and high levels of neighbourhood $\mathrm{NO}_{2}$ concentrations derived from land use regression models; results of these analyses are presented as odds ratios (ORs) with $95 \%$ confidence intervals (Cls).

Results: From 2004 to 2012, 6142 Mls were recorded in Calgary. Individuals living in neighbourhoods with higher long-term air pollution concentrations were more likely to be admitted to hospital for $\mathrm{Ml}$ after short-term elevations in air pollution (e.g., 5-day average $\mathrm{NO}_{2}$ : OR $1.20,95 \% \mathrm{Cl} 1.03-1.40$, per interquartile range [IQR]) as compared with regions with lower air pollution (e.g., 5-day average $\mathrm{NO}_{2}$ : OR 0.90 , 95\% Cl 0.78-1.04, per IQR). In high $\mathrm{NO}_{2}$ tertiles, the AQHI score was associated with $\mathrm{MI}$ (e.g., 5-day average OR 1.13, 95\% Cl 1.02-1.24, per IQR; 3-day average OR 1.13, 95\% Cl 1.04-1.23, per IQR).

Interpretation: Our results show that the effect of air pollution on hospital admissions for $\mathrm{Ml}$ was stronger in areas with higher $\mathrm{NO}_{2}$ concentrations than that in areas with lower $\mathrm{NO}_{2}$ concentrations. Individuals living in neighbourhoods with higher traffic-related pollution should be advised of the health risks and be attentive to special air quality warnings.

tudies have consistently shown that short-term elevations in air pollution concentrations increase the risk of myocardial infarction (MI). ${ }^{1,2}$ Improving our understanding of the effects of short-term exposure to air pollution on MI may inform government policy and facilitate prevention by warning populations at risk. In Calgary, the major contributors to air pollution are transportation for nitrogen dioxide $\left(\mathrm{NO}_{2}\right)$ and carbon monoxide $(\mathrm{CO})$; construction for particulate matter less than $10 \mu \mathrm{m}$ in diameter $\left(\mathrm{PM}_{10}\right)$ and particulate matter less than $2.5 \mu \mathrm{m}$ in diameter $\left(\mathrm{PM}_{2.5}\right)$; and industry for sulfur dioxide $\left(\mathrm{SO}_{2}\right){ }^{3}$ The relatively higher air pollution regions, from a long-term exposure perspective, are mainly distributed along major traffic corridors and close to industrial areas. ${ }^{4,5}$ Air pollution exposure studies that consider an average of air pollution levels overlook the inherent spatial nature of air pollution. , $^{6,7}$
Historically, temporal analyses exploring the association between short-term exposure to air pollution and health outcomes have assumed that pollutants are spatially homogeneous. ${ }^{8-11}$ However, research has shown that spatial distribution patterns differ by pollutant. ${ }^{12}$ For example, it is widely recognized that ozone $\left(\mathrm{O}_{3}\right)$ is relatively spatially homogenous because of consistent concentration levels and temporal fluctuations, whereas $\mathrm{NO}_{2}$ is spatially heterogeneous

Competing interests: See the end of the article.

This article has been peer reviewed.

Correspondence to: Gilaad Kaplan, ggkaplan@ucalgary.ca

CMAJ Open 2020. DOI:10.9778/cmajo.20190160 
because it is attributable to traffic emissions. If city-wide averages are used as air pollution estimates, they fail to consider the spatial variation within a city. ${ }^{11}$ The aim of this study was to determine whether the long-term spatial distribution of air pollution influences the short-term temporal associations between air pollution and MI.

\section{Methods}

\section{Study design}

We used a time-stratified, case-crossover study design to evaluate associations between a short-term exposure and the acute onset of a disease, ${ }^{13,14}$ this is an adaptation of the casecontrol study in which cases serve as their own controls (Figure 1). ${ }^{15}$ This study design has been used extensively to characterize associations between day-to-day changes in air pollution and adverse health events. Because within-individual comparisons are being made, confounding from timeindependent risk factors is controlled for by the design of the study. The case-crossover study design has been shown to effectively control for confounders that are relatively stable in time, such as obesity, diabetes, smoking and socioeconomic status. ${ }^{16}$

The case's exposure at the index time (i.e., day of admission for MI) is compared with its exposure at control time intervals, which are chosen using a time-stratified design. ${ }^{17}$ The index period is measured before the event and the control period is measured before and after the event. ${ }^{18-20}$ For example, if the MI occurs on the second Wednesday in

Temporal analysis: case-crossover study design for the association between daily air pollution levels and $\mathrm{MI}$

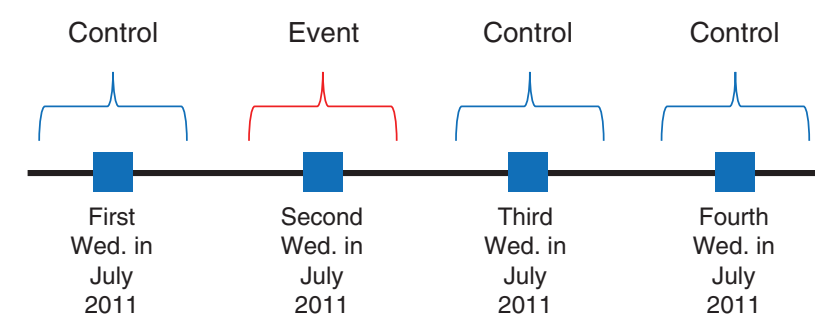

Spatial analysis: case-crossover analysis stratified by low, medium and high nitrogen dioxide levels in Calgary

Total no. of matched risk sets $n=6142$

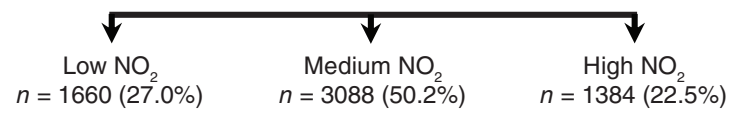

Figure 1: Study design for temporal and spatial analysis of air pollutants effect on hospital admission for myocardial infarction (MI). A time-stratified case-crossover study design that evaluates the shortterm (temporal) effect of $\mathrm{O}_{3}, \mathrm{NO}_{2}, \mathrm{SO}_{2}, \mathrm{CO}, \mathrm{PM}_{10}$ and $\mathrm{PM}_{2.5}$ on $\mathrm{MI}$. Analyses are stratified by high, medium and low levels of $\mathrm{NO}_{2}$ (spatial) as defined by land use regression estimates. Ten patients had missing data. Note: $\mathrm{CO}=$ carbon monoxide, $\mathrm{NO}_{2}=$ nitrogen dioxide, $\mathrm{O}_{3}=$ ozone, $\mathrm{PM}_{10}=$ particulate matter $<10 \mu \mathrm{m}$ in diameter, $\mathrm{PM}_{2.5}=$ particulate matter $<2.5 \mu \mathrm{m}$ in diameter, $\mathrm{SO}_{2}=$ sulfur dioxide. the month of July of 2011, then the referent period will be the other Wednesdays in July of 2011. The time-stratified approach matches the exposure by day of the week and month to control for the influence of day-of-week effects. It also adjusts for seasonal trends in exposure levels. ${ }^{21}$ The timestratified approach is not subject to bias resulting from time trends, because there is no pattern in the placement of referents relative to the index time. ${ }^{16,17,21}$

\section{Study population}

Our population comprised adults over the age of 18 years at the time of incidence of MI, living in Calgary and admitted to hospital with a diagnosis of $\mathrm{MI}$ during the study period from Jan. 1, 2004, to Dec. 31, 2012. Patients who died before presentation to an emergency department were excluded. The population was extracted by first acute MI diagnosis, including ST elevation MI and non-ST elevation MI.

\section{Data sources}

The Alberta Provincial Project for Outcome Assessment in Coronary Heart Disease (APPROACH) is a registry that captures all patients undergoing cardiac catheterization in the province of Alberta since Jan. 1, 1995 (www.approach.org). In 2004, APPROACH expanded to include the Heart Alert initiative in southern Alberta, which enhances data collection by including detailed information on all patients admitted to cardiology services of acute care facilities in Calgary. The data collection is prospective and collected by trained clinical staff using standardized operating procedures and data definitions as part of the medical record in Alberta, and therefore, missing data on key variables are minimal.

Air pollution data were obtained from automated fixed-site continuous monitoring stations maintained by Environment and Climate Change Canada as part of the National Air Pollution Surveillance Network. ${ }^{22-24}$ The 3 stations were Calgary Central, Calgary East and Calgary Northwest, which are positioned to be representative of the background air pollution concentrations across Calgary. Hourly data from each of the 3 fixed sites were averaged together to provide regional estimates of hourly concentrations of the 6 criteria air pollutants investigated in this study: $\mathrm{O}_{3}, \mathrm{NO}_{2}, \mathrm{SO}_{2}, \mathrm{CO}$, $\mathrm{PM}_{10}$ and $\mathrm{PM}_{2.5}$. Daily air pollution levels for Calgary were calculated from hourly records by averaging across the 3 fixed-site monitoring stations. ${ }^{25}$ For all air pollutants, with the exception of ozone, daily mean exposure estimates were used. Ozone values were based on an 8-hour maximum value. In addition, Air Quality Health Index (AQHI) scores were calculated from a composition of 3-hour average values of $\mathrm{O}_{3}, \mathrm{NO}_{2}$ and $\mathrm{PM}_{2.5}$ based on the following formula: ${ }^{26}$

$$
\begin{aligned}
& A Q H I=1000 / 10.4 \times\left[\left(e^{0.000871 \times N 0_{2}}-1\right)+\left(e^{0.000537 \times 0_{3}}-1\right)+\right. \\
& \left.\left(e^{0.000487 \times P M} M_{2.5}-1\right)\right]
\end{aligned}
$$

Data for daily mean temperature and relative humidity were provided by Environment and Climate Change Canada, which averaged the hourly mean temperature and relative humidity across the 3 monitoring stations. These daily time 
series of meteorological data were linked with MI hospital admissions and used as adjustment factors in a multivariable conditional logistic regression model.

Land use regression (LUR) models have been widely used to assess the spatial variation of outdoor air pollution and to estimate fine scale pollution concentrations. ${ }^{27-30}$ Land use regression models capture longer-term measures of ambient air pollution rather than day-to-day fluctuations. Substantial intra-urban variation for $\mathrm{NO}_{2}, \mathrm{PM}_{2.5}$ and metals associated with $\mathrm{PM}_{10}$ has been observed in previous analyses conducted on air pollution with LUR models in Calgary.,31 These previous studies suggest that the major contributors to the spatial variation of air pollution are emissions from motor vehicles and industrial sources, ${ }^{32}$ resulting in relatively higher air pollution along major traffic corridors and the Northeast Industrial area. ${ }^{4,5}$ The stability of LUR models over time has been previously validated. ${ }^{33}$ Further, the LUR model used in Calgary was shown to remain stable over a 5 -year interval. ${ }^{34}$ We used the $\mathrm{NO}_{2}$ estimates from the air pollution study reported by Bertazzon and colleagues for the study period. ${ }^{4}$ Land use regression estimates were assigned to each patient based on the 6-digit postal codes of their residential locations, which we defined as their neighbourhood. Patients who were admitted to hospital for MI were then divided into tertiles based on ambient $\mathrm{NO}_{2}$ concentrations at their residential locations: low $\mathrm{NO}_{2}$ pollution (first tertile), medium $\mathrm{NO}_{2}$ pollution (second tertile) and high $\mathrm{NO}_{2}$ pollution (third tertile) (Figure 2). Patients who were admitted to hospital for MI were assigned to only 1 of the $3 \mathrm{NO}_{2}$ concentrations based on the 6-digit postal codes of their residential locations.

\section{Statistical analysis}

To evaluate the temporal relation between outdoor air pollution levels $\left(\mathrm{O}_{3}, \mathrm{NO}_{2}, \mathrm{SO}_{2}, \mathrm{CO}, \mathrm{PM}_{10}, \mathrm{PM}_{2.5}\right.$ and $\mathrm{AQHI}$ score) and presentation to hospitals because of MI, we constructed several different metrics: same-day exposure, 1-day and 2-day lagged exposures and cumulative 3-day and 5 -day average exposure estimates. Correlation between pollutants was assessed using Pearson correlation coefficients. After matching the case period and referent periods, we used conditional logistic regression to produce risk estimates by comparing exposure data on case and control days. Odds ratios (ORs) with associated 95\% confidence intervals (CIs) were calculated to describe the association between hospital admissions for MI and any increase in the interquartile range (IQR) of the daily concentrations of air pollutants during the different time intervals. We adjusted ORs for temperature and relative humidity. ${ }^{22,35}$ Temperature and relative humidity were entered as linear terms in the models. We verified the linearity of the relation using natural cubic spline functions. The AQHI score was also included in a separate model to explore the composite effects of air pollution on MI. Finally, each pollutant model $\left(\mathrm{O}_{3}, \mathrm{NO}_{2}, \mathrm{SO}_{2}, \mathrm{CO}, \mathrm{PM}_{10}, \mathrm{PM}_{2.5}\right.$ and the $\mathrm{AQHI}$ score) was stratified by an individual's residential exposure to $\mathrm{NO}_{2}$ concentrations (stratified as high, medium and low, based on their 6-digit postal code), as derived from
LUR models. Model stratification of high $\mathrm{NO}_{2}$ was compared with low $\mathrm{NO}_{2}$ concentration using a Cochran $\mathrm{Q}$ test.

\section{Ethics approval}

This study was approved by the University of Calgary's Conjoint Health Research Ethics Board (CHREB) and the Health Canada-Public Health Agency of Canada Research Ethics Board.

\section{Results}

We identified 6142 adult patients admitted to hospital for MI during the study period (Table 1). Of all patients who had an MI, $4482(72.9 \%)$ were men, $3209(52.2 \%)$ patients were aged 65 years or younger, $1493(24.3 \%)$ patients had diabetes and $3646(59.2 \%)$ patients were either a current or former smoker. When stratified by residential location, 1384 (22.5\%) of patients who had an MI lived in neighbourhoods with the highest tertile of $\mathrm{NO}_{2}$ pollution. The distribution of air pollutants and their correlations are provided in Appendix 1, available at www.cmajopen.ca/content/8/4/E619/suppl/DC1.

Associations between short-term air pollution and MI are shown in Table 2. For the overall city-wide study population, only 1-day lag for $\mathrm{SO}_{2}$ exhibited a significant positive association with admissions for MI (OR 1.05, 95\% CI 1.011.09 per IQR). Associations between short-term pollutant concentrations and MI were observed for those residing in neighbourhoods with the highest long-term concentrations of $\mathrm{NO}_{2}$. With the exception of $\mathrm{O}_{3}$, all pollutants were associated with $\mathrm{MI}$ in high $\mathrm{NO}_{2}$ areas with ORs ranging from 1.06 to 1.20 per IQR. The strongest effect on hospital admissions for MI was identified for 5-day cumulative average of $\mathrm{NO}_{2}$ (OR 1.20, 95\% CI 1.03-1.40 per IQR) in high $\mathrm{NO}_{2}$ areas, whereas the association was not significant in low $\mathrm{NO}_{2}$ areas (5-day average $\mathrm{NO}_{2}$ : OR $0.90,95 \%$ CI 0.78-1.04, per IQR; Table 2). In high $\mathrm{NO}_{2}$ regions, the $\mathrm{AQHI}$ was significantly associated with MI (5-day average OR 1.13, 95\% CI 1.021.24 per IQR; 3-day average OR $1.13,95 \%$ CI $1.04-1.23$ per IQR).

\section{Interpretation}

We evaluated the associations between air pollution and risk of hospital admissions for MI with a time-stratified, case-crossover study design. Our analysis was consistent with that of Wang and colleagues, who also explored the effects of air pollution on MI in Calgary. ${ }^{25}$ Neither that study nor ours identified strong temporal effects of air pollution on MI when the spatial distribution of air pollution was assumed to be homogenous across the city of Calgary. The weak association between air pollution and MI in our non-spatially stratified analyses may partially be explained by the generally low air pollution concentrations in Calgary, where warning advisories were issued for less than $1 \%$ of days annually during our study period. ${ }^{36}$ Environment and Climate Change Canada reported in 2015 that air pollution $\left(\mathrm{NO}_{2}, \mathrm{SO}_{2}\right.$, $\mathrm{O}_{3}$ and $\mathrm{CO}$ ) had substantially improved, including in Calgary, from 1990 to $2015 .^{37}$ In a small part, improved air quality in 


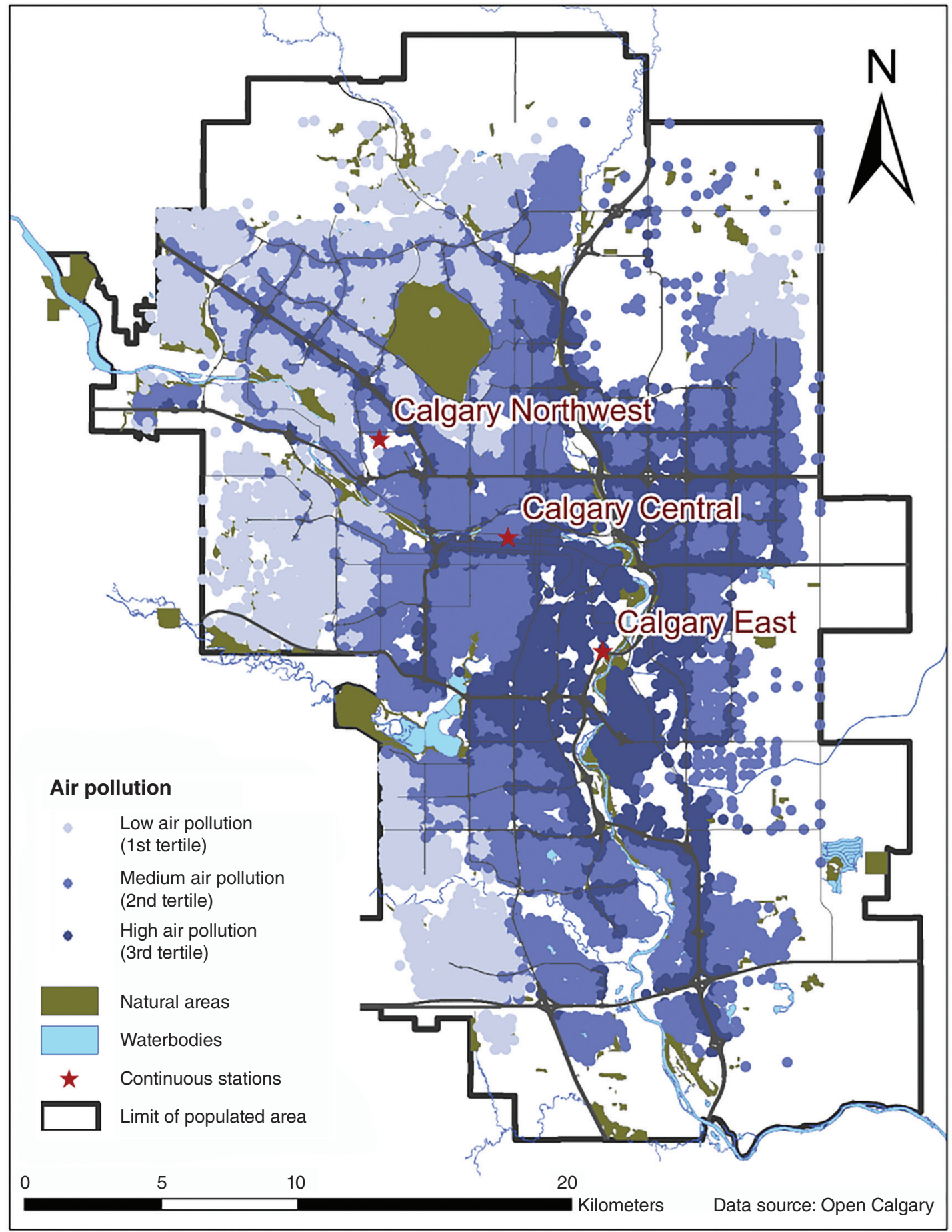

Figure 2: Spatial distribution of nitrogen dioxide in the city of Calgary derived from land use regression estimates. Each dot represents the centroid of a 6-digit postal code with darker shading representing higher air pollution and lighter shading representing lower air pollution. Stars denote the 3 continuous monitoring stations in Calgary.

Calgary may also explain the decreased incidence of hospital admissions for MI in Calgary, as observed by Liu and Bertazzon between 2004 and 2013.38
However, in our study, we also stratified our models by spatial distribution of $\mathrm{NO}_{2}$. Individuals living in regions of high $\mathrm{NO}_{2}$ exposure showed significant associations for all 


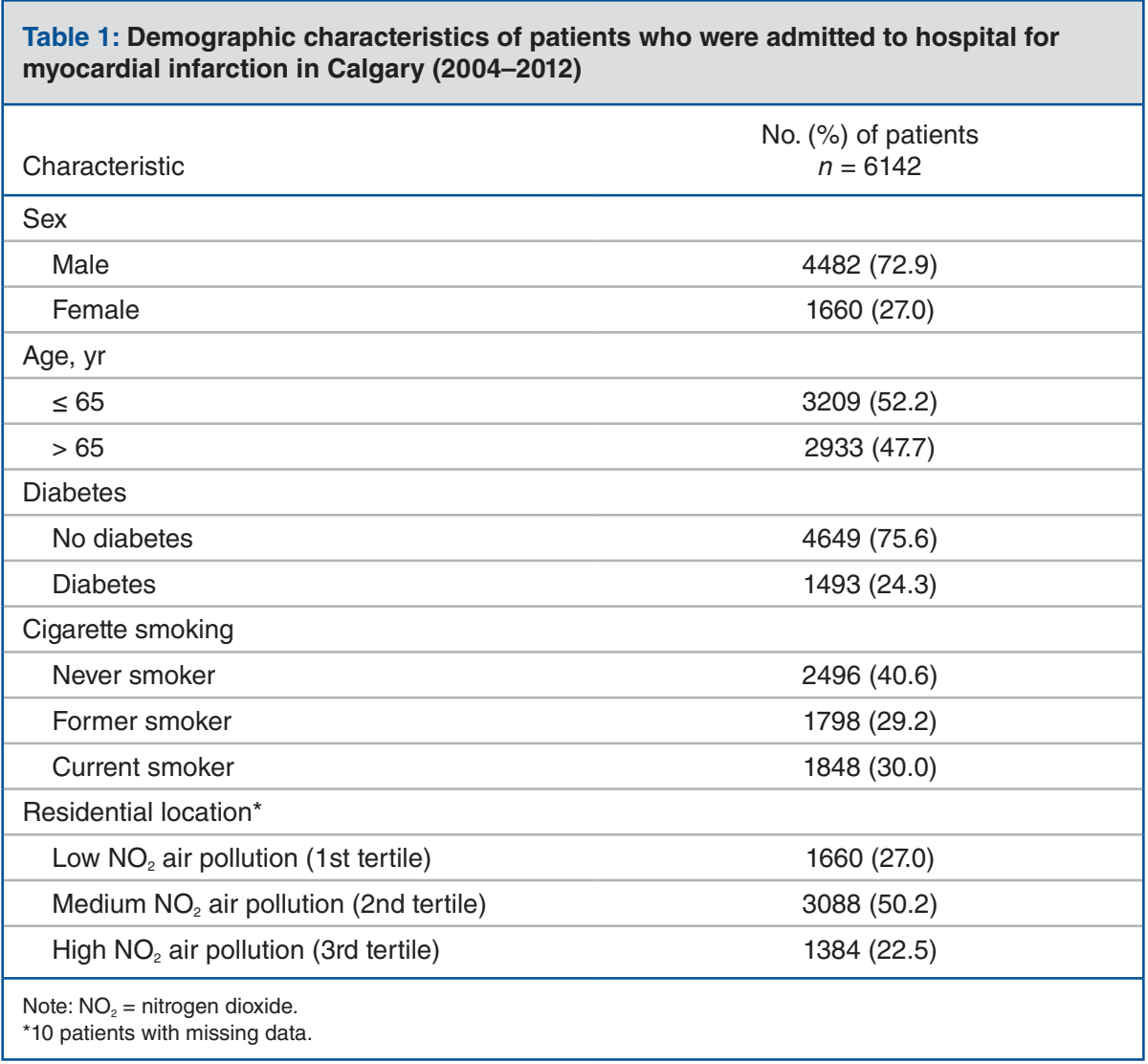

individual pollutants and hospital admissions for MI with the exception of $\mathrm{O}_{3}$. Further, the AQHI score was also associated with hospital admissions for MI for patients living in areas with higher $\mathrm{NO}_{2}$ concentrations. These findings imply that living in a neighbourhood with elevated long-term exposure to $\mathrm{NO}_{2}$ may predispose residents of these areas to the acute effects of air pollution that are associated with an increased risk of MI. Alternatively, acute temporal spikes in air pollution levels across the city may be amplified in areas with higher baseline $\mathrm{NO}_{2}$. These results highlight the importance of accounting for spatial variation when studying the health effects of air pollution.

Associations between $\mathrm{SO}_{2}$ and $\mathrm{MI}$ are consistently reported in the literature. Mustafic and colleagues provide a systematic review concluding that $\mathrm{SO}_{2}$ was positively associated with increased incidence of $\mathrm{MI} .^{2}$ Our results also align with previous studies that report that $\mathrm{O}_{3}$ has no association with hospital admissions for MI. ${ }^{2,25,39}$ In addition, our results suggest that $\mathrm{NO}_{2}$ and $\mathrm{PM}_{2.5}$ levels are associated with increased $\mathrm{MI}$ in areas of medium $\mathrm{NO}_{2}\left(\mathrm{PM}_{2.5}\right.$ only) and areas of high $\mathrm{NO}_{2}$ (both $\mathrm{NO}_{2}$ and $\mathrm{PM}_{2.5}$ ); this is aligned with previous studies that find a positive association between $\mathrm{MI}$ and $\mathrm{NO}_{2}$ and $\mathrm{PM}_{2.5}{ }^{2,35}$

The AQHI score, as a composite score indicating the overall air quality, did not exhibit a positive association with MI except in areas of high $\mathrm{NO}_{2}$. However, most evidence to date indicates that the effects of air pollution are linear, particularly for $\mathrm{O}_{3}$ and $\mathrm{PM}_{2.5}$, such that detection of effects is not dependent on infrequent days with high pollutant concentrations. $^{40,41}$ The AQHI score is calculated based on the combination of $\mathrm{NO}_{2}, \mathrm{O}_{3}$ and $\mathrm{PM}_{2.5}$, of which $\mathrm{O}_{3}$ exhibited no significant associations with $\mathrm{MI}$ among either the entire study population or any subgroups, whereas $\mathrm{NO}_{2}$ and $\mathrm{PM}_{2.5}$ exhibited significant associations in our spatial stratification.

\section{Limitations}

A limitation of our study was the use of fixed-site monitoring data as opposed to personal monitoring. Air pollution studies that rely on outdoor air pollution monitoring are subject to misclassification of the exposure (i.e., measurement error). For example, averaging measurements from the 3 fixed monitoring sites into 1 daily value for the city of Calgary may lead to air pollution exposure misclassification at the individual level. In addition, air pollution monitoring occurred outdoors, which does not account for differences of indoor air pollution exposure. Furthermore, fixed-site monitors do not account for individual mobility, as an individual may not have been near their home when they experienced an MI. The Canadian Human Activity Pattern Survey 2 was a national survey that showed that most individuals spent the day indoors and that seniors spent more than $80 \%$ of their time at or near their home. ${ }^{42}$ Typically, these errors in measurement result in nondifferential exposure misclassification, which serve to underestimate the risks of air pollution..$^{20,43}$

The case-crossover study design of temporal associations controls for non-time dependent confounders; however, factors such as pollen that vary on a daily basis and may be correlated 
Table 2: Association between air pollution and hospital admission for myocardial infarction with increases in the interquartile range of pollutants during various referent time intervals in regions with differing nitrogen dioxide pollution levels

$\mathrm{OR}^{*}(95 \% \mathrm{Cl})$

\begin{tabular}{|c|c|c|c|c|c|}
\hline \multirow[b]{2}{*}{$\begin{array}{l}\text { Pollutant } \\
\text { (median with IQR) }\end{array}$} & \multirow[b]{2}{*}{ Lag (days) } & \multicolumn{4}{|c|}{$\mathrm{OR}^{*}(95 \% \mathrm{Cl})$} \\
\hline & & $\begin{array}{c}\text { Entire study } \\
\text { population } \\
\text { (citywide } \mathrm{NO}_{2} \text { ) } \\
n=6142\end{array}$ & $\begin{array}{l}\text { Low } \mathrm{NO}_{2} \text { tertile } \\
\qquad n=1660\end{array}$ & $\begin{array}{l}\text { Medium } \mathrm{NO}_{2} \text { tertile } \\
\quad n=3088\end{array}$ & $\begin{array}{c}\text { High } \mathrm{NO}_{2} \text { tertile } \\
n=1384\end{array}$ \\
\hline \multirow{5}{*}{$\begin{array}{l}\mathrm{CO} \\
(0.35, \text { IQR } 0.27-0.47)\end{array}$} & 0 index day & $0.97(0.93-1.02)$ & $0.94(0.88-1.01)$ & $0.97(0.92-1.02)$ & $1.02(0.95-1.09)$ \\
\hline & 1-day lag & $1.03(0.98-1.08)$ & $0.98(0.92-1.05)$ & $1.02(0.97-1.07)$ & $1.10(1.02-1.18)$ \\
\hline & 2-day lag & $1.01(0.96-1.06)$ & $1.03(0.96-1.10)$ & $0.99(0.94-1.04)$ & $1.04(0.97-1.12)$ \\
\hline & 0- to 2-day average & $1.01(0.94-1.08)$ & $0.97(0.88-1.06)$ & $0.99(0.92-1.05)$ & $1.09(0.99-1.21)$ \\
\hline & 0 - to 4-day average & $0.97(0.90-1.05)$ & $0.92(0.83-1.03)$ & $0.96(0.88-1.03)$ & $1.08(0.96-1.21)$ \\
\hline \multirow{5}{*}{$\begin{array}{l}\mathrm{NO}_{2} \\
(18.22, \text { IQR 12.67-25.00) }\end{array}$} & 0 index day & $1.00(0.94-1.07)$ & $0.97(0.89-1.06)$ & $1.01(0.95-1.08)$ & $1.05(0.95-1.15)$ \\
\hline & 1-day lag & $1.04(0.97-1.11)$ & $1.00(0.91-1.09)$ & $1.01(0.94-1.07)$ & $1.16(1.05-1.28) \dagger$ \\
\hline & 2-day lag & $1.03(0.97-1.10)$ & $1.03(0.94-1.12)$ & $1.00(0.93-1.06)$ & $1.11(1.01-1.22)$ \\
\hline & 0- to 2-day average & $1.05(0.96-1.14)$ & $1.00(0.88-1.12)$ & $1.01(0.92-1.10)$ & $1.20(1.05-1.36)$ \\
\hline & 0- to 4-day average & $0.98(0.88-1.08)$ & $0.90(0.78-1.04)$ & $0.93(0.84-1.03)$ & $1.20(1.03-1.40) \dagger$ \\
\hline \multirow{5}{*}{$\begin{array}{l}\mathrm{O}_{3} \max \\
(39.00, \text { IQR } 32.00-47.00)\end{array}$} & 0 index day & $1.00(0.95-1.06)$ & $1.02(0.96-1.10)$ & $0.98(0.93-1.03)$ & $1.03(0.96-1.11)$ \\
\hline & 1-day lag & $0.99(0.94-1.04)$ & $0.96(0.90-1.03)$ & $0.97(0.93-1.03)$ & $1.07(0.99-1.15)$ \\
\hline & 2-day lag & $0.99(0.94-1.04)$ & $0.97(0.91-1.04)$ & $0.99(0.94-1.04)$ & $1.02(0.94-1.10)$ \\
\hline & 0- to 2-day average & $0.99(0.93-1.06)$ & $0.98(0.90-1.07)$ & $0.97(0.91-1.03)$ & $1.06(0.96-1.17)$ \\
\hline & 0- to 4-day average & $1.00(0.93-1.08)$ & $1.00(0.90-1.11)$ & $0.97(0.90-1.04)$ & $1.09(0.97-1.22)$ \\
\hline \multirow{5}{*}{$\begin{array}{l}\mathrm{SO}_{2} \\
(1.00, \text { IQR } 1.00-2.00)\end{array}$} & 0 index day & $1.00(0.96-1.05)$ & $1.00(0.95-1.06)$ & $0.97(0.93-1.01)$ & $1.08(1.02-1.15) \dagger$ \\
\hline & 1-day lag & 1.05 (1.01-1.09) & $1.03(0.98-1.09)$ & $1.04(1.00-1.08)$ & $1.10(1.03-1.16)$ \\
\hline & 2-day lag & $1.04(0.99-1.08)$ & $1.04(0.98-1.10)$ & $1.03(0.99-1.07)$ & $1.05(0.99-1.12)$ \\
\hline & 0- to 2-day average & $1.06(1.00-1.12)$ & $1.05(0.97-1.14)$ & $1.03(0.97-1.09)$ & $1.15(1.06-1.25)$ \\
\hline & 0 - to 4-day average & $1.05(0.98-1.12)$ & $1.05(0.96-1.16)$ & $1.02(0.95-1.09)$ & $1.10(0.99-1.22)$ \\
\hline \multirow{5}{*}{$\begin{array}{l}\mathrm{PM}_{10} \\
20.00, \text { IQR } 14.00-30.00)\end{array}$} & 0 index day & $0.98(0.95-1.02)$ & $0.95(0.90-1.00)$ & $0.98(0.95-1.02)$ & $1.03(0.97-1.08)$ \\
\hline & 1-day lag & $1.01(0.97-1.05)$ & $0.97(0.92-1.02)$ & $1.01(0.97-1.04)$ & $1.06(1.01-1.12) \dagger$ \\
\hline & 2-day lag & $1.01(0.98-1.05)$ & $0.99(0.95-1.04)$ & $1.00(0.96-1.04)$ & $1.06(1.00-1.12)$ \\
\hline & 0- to 2-day average & $1.00(0.96-1.05)$ & $0.95(0.89-1.01)$ & $0.99(0.95-1.04)$ & $1.08(1.01-1.16) \dagger$ \\
\hline & 0 - to 4-day average & $0.99(0.94-1.05)$ & $0.95(0.88-1.02)$ & $0.98(0.93-1.04)$ & $1.07(0.99-1.15)$ \\
\hline \multirow{5}{*}{$\begin{array}{l}\mathrm{PM}_{2.5} \\
(7.00, \mathrm{IQR} 4.33-10.50)\end{array}$} & 0 index day & $1.01(0.98-1.05)$ & $0.99(0.94-1.05)$ & $1.00(0.97-1.04)$ & $1.06(1.00-1.11)$ \\
\hline & 1-day lag & $1.02(0.99-1.06)$ & $0.98(0.93-1.03)$ & $1.04(1.01-1.08)$ & $1.04(0.99-1.10)$ \\
\hline & 2-day lag & $1.00(0.96-1.04)$ & $0.96(0.90-1.01)$ & $1.02(0.97-1.06)$ & $1.03(0.98-1.08)$ \\
\hline & 0- to 2-day average & $1.02(0.97-1.07)$ & $0.96(0.90-1.03)$ & $1.03(0.98-1.08)$ & $1.07(1.00-1.13)$ \\
\hline & 0- to 4-day average & $1.02(0.97-1.08)$ & $0.98(0.91-1.06)$ & $1.04(0.99-1.10)$ & $1.04(0.96-1.12)$ \\
\hline \multirow{5}{*}{$\begin{array}{l}\text { AQHI } \\
(4.01, \text { IQR 3.49-4.65) }\end{array}$} & 0 index day & $1.01(0.96-1.05)$ & $1.00(0.94-1.06)$ & $0.99(0.95-1.04)$ & $1.06(0.99-1.13)$ \\
\hline & 1-day lag & $1.02(0.97-1.07)$ & $0.97(0.91-1.03)$ & $1.00(0.96-1.05)$ & $1.12(1.05-1.20) \dagger$ \\
\hline & 2-day lag & $1.01(0.97-1.06)$ & $0.99(0.93-1.05)$ & $1.00(0.95-1.04)$ & $1.07(1.00-1.14)$ \\
\hline & 0- to 2-day average & $1.02(0.96-1.08)$ & $0.98(0.90-1.06)$ & $1.00(0.94-1.05)$ & $1.13(1.04-1.23) \dagger$ \\
\hline & 0 - to 4-day average & $1.01(0.94-1.08)$ & $0.97(0.89-1.07)$ & $0.97(0.91-1.04)$ & $1.13(1.02-1.24) \dagger$ \\
\hline
\end{tabular}

Note: $\mathrm{Cl}=$ confidence interval, $\mathrm{CO}=$ carbon monoxide, $\mathrm{IQR}=$ interquartile range, $\mathrm{NO}_{2}=$ nitrogen dioxide, $\mathrm{OR}=$ odds ratio, $\mathrm{O}_{3}=\mathrm{ozone}, \mathrm{PM}{ }_{10}=$ particulate matter $<10 \mu \mathrm{m}$ in diameter, $\mathrm{PM}_{2.5}=$ particulate matter $<2.5 \mu \mathrm{m}$ in diameter, $\mathrm{SO}_{2}=$ sulfur dioxide.

*Odds ratios are adjusted for temperature and relative humidity.

†Significant difference comparing $\mathrm{OR}$ in the highest $\mathrm{NO}_{2}$ tertile to the lowest $\mathrm{NO}_{2}$ tertile using the Cochran $\mathrm{Q}$ test.

Significant associations are bolded. 
to air pollution levels are not controlled in a case-crossover study design. Although temporally stable cardiac risk factors (e.g., hypertension, diabetes and dyslipidemia) are controlled, risk factors such as smoking that may vary from day to day (e.g., smoking only on the weekend) may introduce bias in the temporal analyses. In addition, our stratified spatial analysis based on residential $\mathrm{NO}_{2}$ exposure was subject to confounding because high pollution areas may correspond with other risk factors for MI, such as low socioeconomic status and obesity. For example, an individual with low socioeconomic status may live in proximity to major traffic arteries (i.e., higher air pollution) because these neighbourhoods have lower property value. Thus, the results of the current study should be interpreted cautiously without inference to causality. Further investigation on whether living in a high pollution area increases vulnerability to temporal spikes in pollution concentrations is necessary.

Misclassification of the timing of onset of MI may introduce bias into the results. In addition, the study was restricted to individuals who were admitted to hospital with MI; the study did not account for individuals who died out of hospital. Multiple comparison errors may account for some of the significant associations observed and pollutants are often correlated, leading to lack of independence, and thus, replication studies are necessary.

\section{Conclusion}

We evaluated the effects of increased air pollution on the increased odds of hospital admissions for MI by integrating spatial variation in air pollution derived from $\mathrm{NO}_{2}$ LUR models. Our results show that the effect of air pollution on $\mathrm{MI}$ was stronger in areas with higher $\mathrm{NO}_{2}$ concentrations than that in areas with lower $\mathrm{NO}_{2}$ concentrations. These results highlight the need for preventive strategies targeted specifically to populations living in residential areas with higher traffic-related pollution, who should be advised of the health risks and to pay particular attention to special air quality statements.

\section{References}

1. Dominici F, Peng RD, Bell ML, et al. Fine particulate air pollution and hospital admission for cardiovascular and respiratory diseases. FAMA 2006;295:1127-34.

2. Mustafic H, Jabre P, Caussin C, et al. Main air pollutants and myocardial infarction. 7AMA 2012;307:713-21.

3. All source pollutant emissions inventory for the Calgary Region Airshed Zone (CRAZ) spatial allocation project (2012-2013) (final draft). Calgary: Calgary Region Airshed Zone; 2013.

4. Bertazzon S, Johnson M, Eccles K, et al. Accounting for spatial effects in land use regression for urban air pollution modeling. Spat Spatiotemporal Epidemiol 2015;14-15:9-21.

5. Liu X, Bertazzon S. Fine scale spatio-temporal modelling of urban air pollution. In: Miller AJ, O'Sullivan D, Wiegand N, editors. Proceedings from Geographic Information Science: 9th International conference of GIScience 2016. [lecture notes in computer science, vol 9927]. Sept. 27-30; Montreal. Cham (Switzerland): Springer International Publishing; 2016:210-24. Available: https://doi. org/10.1007/978-3-319-45738-3_14 (accessed 2019 July 25).

6. Jerrett M, Burnett RT, Ma R, et al. Spatial analysis of air pollution and mortality in Los Angeles. Epidemiology 2005;16:727-36.

7. Basagaña X, Aguilera I, Rivera M, et al. Measurement error in epidemiologic studies of air pollution based on land-use regression models. Am 7 Epidemiol 2013;178:1342-6.

8. D'Ippoliti D, Forastiere F, Ancona C, et al. Air pollution and myocardial infarction in Rome: a case-crossover analysis. Epidemiology 2003;14:528-35.

9. Ruidavets JB, Cournot M, Cassadou S, et al. Ozone air pollution is associated with acute myocardial infarction. Circulation 2005;111:563-9.
10. Lanki T, Pekkanen J, Aalto P, et al. Associations of traffic related air pollutants with hospitalisation for first acute myocardial infarction: the HEAPSS study. Occup Environ Med 2006;63:844-51.

11. Gryparis A, Paciorek CJ, Zeka A, et al. Measurement error caused by spatial misalignment in environmental epidemiology. Biostatistics 2009;10:258-74.

12. Sarnat SE, Klein M, Sarnat J, et al. An examination of exposure measurement error from air pollutant spatial variability in time-series studies. 7 Expo Sci Environ Epidemiol 2010;20:135-46.

13. Peters A, Dockery DW, Muller JE, et al. Increase particulate air pollution and the triggering of myocardial infarction. Circulation 2001;103:2810-5.

14. Rich DQ, Schwartz J, Mittleman MA, et al. Association of short-term ambient air pollution concentrations and ventricular arrhythmias. Am 7 Epidemiol 2005;161:1123-32.

15. Maclure M. The case-crossover design: a method for studying transient effects on the risk of acute events. Am 7 Epidemiol 1991;133:144-53.

16. Schwartz J. The effects of particulate air pollution on daily deaths: a multicity case crossover analysis. Occup Environ Med 2004;61:956-61.

17. Janes H, Sheppard L, Lumley T. Case-crossover analyses of air pollution exposure data: Referent selection strategies and their implications for bias. Epidemiology 2005;16:717-26.

18. Bateson TF, Schwartz J. Selection bias and confounding in case-crossover analysis of environmental time-series data. Epidemiology 2001;12:654-61.

19. Navidi W, Weinhandl E. Risk set sampling for case-crossover designs. Epidemiology 2002;13:100-5.

20. Jaakkola JJK. Case-crossover design in air pollution epidemiology. Eur Respir Z Suppl 2003;40:81s-5s.

21. Levy D, Lumley T, Sheppard L, et al. Referent selection in case-crossover analyses of acute health effects of air pollution. Epidemiology 2001;12:186-92.

22. Kaplan GG, Dixon E, Panaccione R, et al. Effect of ambient air pollution on the incidence of appendicitis. CMA7 2009;181:591-7.

23. Kaplan GG, Tanyingoh D, Dixon E, et al. Ambient ozone concentrations and the risk of perforated and nonperforated appendicitis: a multicity casecrossover study. Environ Health Perspect 2013;121:939-43.

24. National Air Pollution Surveillance (NAPS) Network. Ottawa: Government of Canada, National Air Pollution Surveillance; 2018. Available: https://open. canada.ca/data/en/dataset/1b36a356-defd-4813-acea-47bc3abd859b (accessed 2019 July 25).

25. Wang X, Kindzierski W, Kaul P, et al. Air pollution and acute myocardial infarction hospital admission in Alberta, Canada: a three-step procedure case-crossover study. PLoS One 2015;10:e0132769. doi: 10.1371/journal. pone.0132769.

26. Stieb DM, Burnett RT, Smith-Doiron M, et al. A new multipollutant, nothreshold air quality health index based on short-term associations observed in daily time- series analyses. 7 Air Waste Manag Assoc 2008;58:435-50.

27. Ryan PH, LeMasters GK. A review of land-use regression models for characterizing intraurban air pollution exposure. Inhal Toxicol 2007;19(Suppl 1):127-33.

28. Hoek G, Beelen R, de Hoogh K, et al. A review of land-use regression models to assess spatial variation of outdoor air pollution. Atmos Environ 2008; 42:7561-78.

29. Mercer LD, Szpiro AA, Sheppard L, et al. Comparing universal kriging and land-use regression for predicting concentrations of gaseous oxides of nitrogen (NOx) for the Multi-Ethnic Study of Atherosclerosis and Air Pollution (MESA Air). Atmos Environ (1994) 2011;45:4412-20.

30. Wang M, Beelen R, Eeftens M, et al. Systematic evaluation of land use regression models for $\mathrm{NO}_{2}$. Environ Sci Technol 2012;46:4481-9.

31. Zhang JJY, Sun L, Barrett O, et al. Development of land-use regression models for metals associated with airborne particulate matter in a North American city. Atmos Environ 2015;106:165-77.

32. Calgary Region Airshed Zone (CRAZ) 2013 annual report. Calgary: Calgary Region Airshed Zone; 2013

33. Wang R, Henderson SB, Sbihi H, et al. Temporal stability of land use regression models for traffic-related air pollution. Atmos Environ 2013;64: 312-9.

34. Bertazzon S, Couloigner I, Underwood FE. Spatial land use regression of nitrogen dioxide over a 5-year interval in Calgary, Canada. Int $\mathcal{f}$ GIS 2019; 33:1335-54.

35. Bhaskaran K, Hajat S, Armstrong B, et al. The effects of hourly differences in air pollution on the risk of myocardial infarction: case crossover analysis of the MINAP database. BM7 2011;343:d5531.

36. Daley $\mathrm{S}$. Analysis of the air quality health index in Canadian urban centres. Ottawa: Environment and Climate Change Canada; 2010. Available: https://portal.cfpc. ca/resourcesdocs/uploadedFiles/Directories/_PDFs/AQHI_Analysis_2001-2009. pdf (accessed 2019 July 25).

37. Air pollution emission inventory report. Ottawa: Environment and Climate Change Canada; 2015.

38. Liu X, Bertazzon S. Exploratory temporal and spatial analysis of myocardial infarction hospitalizations in Calgary, Canada. Int 7 Environ Res Public Health 2017;14:1555.

39. Vidale S, Arnaboldi M, Bosio V, et al. Short-term air pollution exposure and cardiovascular events: a 10-year study in the urban area of Como, Italy. Int $\mathcal{F}$ Cardiol 2017;248:389-93. 
40. Newell K, Kartsonaki C, Lam KBH, et al. Cardiorespiratory health effects of gaseous ambient air pollution exposure in low- and middle-income countries: a systematic review and meta-analysis. Environ Health 2018;17:41.

41. WHO air quality guidelines for particulate matter, ozone, nitrogen dioxide and sulfur dioxide: global update 2005: summary of risk assessment. Geneva: World Health Organization; 2006. Available: www.who.int/phe/health_topics/outdoorair/ outdoorair_aqg/en/ (accessed 2019 Sept. 17).

42. Matz CJ, Stieb DM, Davis K, et al. Effects of age, season, gender and urbanrural status on time-activity: Canadian Human Activity Pattern Survey 2 (CHAPS 2). Int 7 Environ Res Public Health 2014;11:2108-24.

43. Bell ML, Peng RD, Dominici F. The exposure-response curve for ozone and risk of mortality and the adequacy of current ozone regulations. Environ Health Perspect 2006;114:532-6.

Competing interests: Michael Hill reports personal fees from Sun Pharma; nonfinancial support from Hoffmann-La Roche Canada; grants from Covidien (Medtronic), Boehringer-Ingelheim, Stryker, Medtronic and NoNO; and the patent "Systems and Methods for Assisting in Decision-Making and Triaging for Acute Stroke Patients." Michael Hill owns stock in Calgary Scientific, a company that focuses on medical imaging software, is a director of the Canadian Neurological Sciences Federation, a not-for-profit group, is a director of Circle NeuroVascular, and has received grant support from Alberta Innovates Health Solutions, Canadian Institutes of Health Research, Heart and Stroke Foundation of Canada, and the National Institute of Neurological Disorders and Stroke. Gilaad Kaplan has received honoraria for speaking or consultancy from AbbVie, Janssen, Pfizer and Takeda. He has received research support from Ferring, Janssen, AbbVie, GlaxoSmithKline, Merck and Shire. He has been a consultant for Gilead. He shares ownership of a patent: Treatment of Inflammatory Disorders, Autoimmune Disease, and PBC. UTI Limited Partnership, assignee. Patent WO2019046959A1. PCT/ CA2018/051098. 7 Sept. 2018. No other competing interests were declared.

Affiliations: Departments of Community Health Sciences (Liu, Coward, Tanyingoh, Windsor, Underwood, Rabi, Ghali, James, Kaplan) and of Geography (Liu, Bertazzon), University of Calgary, Calgary, Alta.; Department of History, Archaeology, Geography, Fine \& Performing Arts (Bertazzon), University of Florence, Florence, Italy; School of Mathematics and Statistics and Department of Neuroscience, and CHAIM Research Centre (Villeneuve), Carleton University, Ottawa, Ont.; Air Health Science Division (Johnson), Health Canada, Ottawa, Ont.; Environmental Health Science and Research Bureau (Stieb), Health Canada, Vancouver, BC;
Departments of Medicine (Coward, Tanyingoh, Windsor, Underwood, Hill, Rabi, Wilton, James, Kaplan); of Clinical Neurosciences (Hill, Ghali); and of Cardiac Sciences (Wilton), University of Calgary, Calgary, Alta.; Department of Medicine (Graham, McMurtry), University of Alberta; Mazankowski Alberta Heart Institute (McMurtry), Edmonton, Alta.

Contributors: Xiaoxiao Liu, Stefania Bertazzon, Paul Villeneuve, Markey Johnson, Dave Stieb, Michael Hill, Doreen Rabi, Michelle Graham, M. Sean McMurtry and Gilaad Kaplan contributed to study design. Xiaoxiao Liu, Stefania Bertazzon, Markey Johnson, Doreen Rabi, Michael Hill, William Ghali, Stephen Wilton, Matthew James and Gilaad Kaplan contributed to data collection. Xiaoxiao Liu, Stefania Bertazzon, Paul Villeneuve, Markey Johnson, Stephanie Coward, Divine Tanyingoh and Gilaad Kaplan contributed to data analysis. Xiaoxiao Liu, Stefania Bertazzon, Paul J. Villeneuve, Markey Johnson, Dave Stieb, Stephanie Coward, Divine Tanyingoh, Michael Hill, Doreen Rabi, William Ghali, Stephen Wilton, Matthew James, Michelle Graham, M. Sean McMurtry, Joseph Windsor, Fox Underwood and Gilaad Kaplan interpreted data and all authors wrote or edited the manuscript. Gilaad Kaplan had full access to all of the data in the study and takes responsibility for the integrity of the data and the accuracy of the data analysis. All of the authors gave final approval of the version to be published and agreed to be accountable for all aspects of the work.

Data sharing: Health data cannot be shared publicly because of restrictions regarding patient privacy. To request access to data from the APPROACH database, visit the website at https://www.approach.org/ contact_pages/contact.html. Air pollution data from the National Air Pollution Surveillance (NAPS) program is open access and can be accessed at this site: https://open.canada.ca/data/en/dataset/1b36a356 -defd-4813-acea-47bc3abd859b.

Acknowledgment: Gilaad Kaplan is a CIHR Embedded Clinician Research Chair.

Funding: This study was funded by a Canadian Institutes of Health Research Operating Grant (Funding Reference Number 133485) and by Health Canada.

Supplemental information: For reviewer comments and the original submission of this manuscript, please see www.cmajopen.ca/content/8/4/ E619/suppl/DC1. 